\title{
CLASSICAL RELATIVISTIC EQUATIONS FOR PARTICLES WITH SPIN MOVING IN EXTERNAL FIELDS
}

\author{
H. VAN DAM* and Th.W. RUIJGROK \\ Instituut voor Theoretische Fysica, Utrecht, The Netherlands**
}

Received 8 August 1980

\begin{abstract}
We derive equations of motion for a point particle with spin in an external electromagnetic and in an external scalar field. The derivation is based on the ten conservation laws of linear and angular momentum and on a general expression for the current by which the particle interacts with the external fields.
\end{abstract}

\section{Introduction and summary}

In this paper we want to discuss the old problem of how a classical, but relativistic particle with spin moves through an inhomogeneous external field.

Our motivation for studying this problem is not to prepare the stage for quantization-after all, the Dirac equation is good enough-but rather to derive the most general equations of motion, which are consistent with a number of "reasonable" conditions. The belief that such equations exist is based on the assumption that a classical particle with a macroscopic spin is a valid concept and need not be related to a quantum theory. A large number of authors sharing this belief is quoted in ref. 1-3. To be complete we must, however, mention that Bohr showed that a classical description can only be correct if the magnetic moment of the particle is large compared with $e h / m c^{4}$ ). We also want to mention explicitly the experimental use of the BargmannMichel-Telegdi equation ${ }^{5}$ ) in determining the $g$ factors of the electron and muon. These equations, however, neglect gradients of the electromagnetic field and do not include the Stern-Gerlach effect, as we shall see later. There have been arguments that the Stern-Gerlach effect is not observable for electrons ${ }^{6}$ ), as distinguished from atoms. This may be true because the mass of the electrons is small. Here, however, we consider particles with arbitrary mass and then it is legitimate to ask for a relativistic description which includes the effects of field gradients.

*On leave from the University of North Carolina, Chapel Hill N.C., USA.

**Postal address: Princetonplein 5, P.O. Box 80006, 3508 TA Utrecht, The Netherlands. 
We now discuss the conditions on our equations which we consider reasonable, as well as some questions of notation. We shall use the metric $-g_{00}=g_{11}=g_{22}=g_{33}=1$, and choose the velocity of light, $c$, to be 1 . As the parameter along the worldline we shall use proper time, $\tau$, although, in order to prevent inconsistencies, it is necessary to do this with care. In particular one must make this choice only after obtaining the equations of motion by varying, for instance, an action, as we shall see in an example in section 2 . Defining

$$
\begin{aligned}
& u^{\mu}=\frac{\mathrm{d}}{\mathrm{d} \tau} X^{\mu}(\tau), \quad \text { we have with } \tau \text { the proper time: } \\
& u^{2}=u^{\mu} u_{\mu}=-1 .
\end{aligned}
$$

For spinless particles, in which case we have a Lagrangian available, $p^{\mu}$ is parallel to $u^{\mu}$ because in the particle part of the Lagrangian Poincaré invariance allows $\dot{x}^{2}$ as the only scalar available. By $p^{\mu}$ we mean the particle momentum which e.g. for a point particle without spin in an electromagnetic field is given by

$$
p_{\mu}=\frac{\partial \mathscr{L}}{\partial \dot{X}^{\mu}}-e A_{\mu}
$$

For particles with spin, however, $\dot{x}=u$ can be contracted into a scalar with the internal variables related to the spin. Thus, assuming we had a Lagrangian, then $p$ and $u$ will in general not be parallel for a particle with spin.

Calling (1.1) our first condition, our second condition is that $p$ and $u$ must be parallel in regions where the external fields are zero. This second condition excludes helical motions which free particles may have in some theories $\left.{ }^{8}\right)$. As $p$ is constant in field free regions, condition number two implies that the world line is straight in these regions.

Our third condition is related to the spin, $\sigma^{\alpha \beta}(\tau)=-\sigma^{\beta \alpha}(\tau)$. We know that for free particles one should have

$$
\sigma_{\alpha \beta}(\tau) u^{\beta}(\tau)=0,
$$

i.e. the spin is purely spacelike in the rest frame of the particle. It is natural to assume (1.2a) also in regions where the external field is not zero $)$. This assumption, however, leads to theories which do not satisfy our second condition, therefore we consider an alternative possibility')

$$
\sigma_{\alpha \beta}(\tau) p^{\beta}(\tau)=0 .
$$

With our second condition $(1.2 b)$ reduces to $(1.2 a)$ in regions with zero external field. In section 2 we shall investigate both possibilities (1.2a) and (1.2b) for a scalar field. We then show that (1.2a) combined with our fourth 
and main condition leads to inacceptable theories of the type found by Frenkel ${ }^{8}$ ).

Our fourth condition is that the equation of motion be compatible with the ten conservation laws of linear momentum and of angular momentum (both in the four-dimensional Minkowski sense) of particle plus field.

As our fifth condition we ask that the equation of motion contain time derivatives of the position of no higher than second order, and time derivatives of the spin of no higher than first order.

In sections 2 and 3, proceeding on the basis of the first four conditions, we shall find that two more desirable conditions are satisfied: condition six

$$
\frac{\mathrm{d}}{\mathrm{d} \tau} p^{2}=0, \text { or } \quad p^{2}=-m^{2}=\text { constant }
$$

and condition seven

$$
\frac{\mathrm{d}}{\mathrm{d} \tau}\left(\sigma^{\mu \nu} \sigma_{\mu \nu}\right)=\mathbf{0}
$$

For the scalar field, and for the electromagnetic field with zero gyromagnetic interaction (1.3) and (1.4) are satisfied exactly. For the general electromagnetic field (1.4) holds again but the condition (1.3) is only satisfied up to second order in the external fields. We will therefore restrict ourselves to equations which only linearly depend on the fields and their derivatives, so that no particles with quadrupole moments are admitted.

To be complete, let us mention the alternative description of spin by a four vector $W^{\mu}$, the so-called "Paul-Lubański" vector. In order that this vector $W^{\mu}$ contains the same information as $\sigma^{\mu \nu}$, its definition depends on whether one has (1.2a) or (1.2b). In the first case one has

$$
W^{\mu}=\frac{1}{2} \epsilon^{\mu \nu \lambda \sigma} \sigma_{\nu \lambda} u_{\sigma}
$$

and

$$
\sigma_{\alpha \beta}=\epsilon_{\alpha \beta \mu \nu} W^{\mu} u^{\nu}
$$

In the second case one needs instead

$$
\begin{aligned}
W^{\mu} & =\frac{1}{2 m} \epsilon^{\mu \nu \lambda \sigma} \sigma_{\nu \lambda} p_{\sigma}, \\
\sigma_{\alpha \beta} & =\frac{1}{m} \epsilon_{\alpha \beta \mu \nu} W^{\mu} p^{\nu} .
\end{aligned}
$$

The Lagrangian description, which is available for spinless particles, is used in what follows as a heuristic tool. Actually several examples of Lagrangians for spinning particles are available ${ }^{7}$ ), but they all seem to have disadvantages, 
as discussed by Grassberger ${ }^{10}$ ). One thing which we take over from the Lagrangian description is the requirement of "chronometric invariance""). This requirement stipulates that the theory be invariant under a change in the parameter labelling the events along the world line of the particle. This parameter can then afterwards be chosen in such a way that the acceleration in the direction of the velocity vanishes: $\dot{u} \cdot u=0$, i.e. this insures our first condition. This chronometric invariance is helpful in the case of the scalar field and also in finding the correct gyromagnetic interaction with the Maxwell field.

We will assume that there is a Lagrangian density for the external fields including the source terms describing the actions of the particle on the field. Thus, we can derive that part of the energy-momentum tensor which contains contributions from the field and from the interaction with the particle, but not from the particle alone. We will then impose the conservation laws, which then provide the equations of motion for the particle and its spin, after we have decided what the relation is between the momentum and the velocity. This relation is fixed by the "reasonable" conditions, which we mentioned before.

In the next section we derive the equations for a particle with spin moving in a scalar external field. The result we obtain is a generalization of the equations given by Schwinger ${ }^{11}$ ) in that also derivatives of the field now occur. In section 3 the method is applied to the electromagnetic field. Our final equations are the same as those given by Suttorp and de Groot ${ }^{1}$ ) (eqs. (61) and (62) of ref. 1). They have an extra term as compared to the equations of $\mathrm{Good}^{12}$ ). For a homogeneous field our equations reduce to those of $B M T^{5}$ ), but for an inhomogeneous field we also find a so-called Stern-Gerlach term.

Despite the fact that our final equations are identical to those of Suttorp and de Groot, our derivation of these equations has a number of advantages. These will be discussed at the end of section 3 .

Our approach, further, is of great generality and avoids, among other things, difficulties in the Lagrangian method connected with the difference in center of energy and center of charge.

\section{Spinning particle in a scalar field}

In this section we want to discuss the derivation of the equations of motion for a particle with spin, moving in an external scalar field $\phi(x)$. We will first obtain the equations for a spinless particle from a Lagrangian formulation and then show how the same equations can be derived from the conservation law 
for energy-momentum. This second method is then applied to the case of a particle with spin.

Consider the action functional of the field $\phi(x)$ and of the world line $X(\tau)$ of the particle

$$
A=\int \mathrm{d}_{4} x \sqrt{\tilde{g}} \mathscr{L},
$$

where

$$
\tilde{g}=-\operatorname{det}\left\|g_{\mu \nu}\right\|=+1
$$

and the Lagrangian density is given by

$$
\mathscr{L}=-\frac{1}{2} g^{\mu \nu} E_{\mu} E_{\nu}-\int \mathrm{d} \tau \tilde{g}^{-1 / 2}(m-e \phi(x)) \delta_{4}(x-X(\tau)) \sqrt{-g_{\alpha \beta} u^{\alpha} u^{\beta}} .
$$

We have introduced the vector field and the 4-velocity by

$$
E_{\mu}=\partial_{\mu} \phi \quad \text { and } \quad u^{\alpha}=\frac{\mathrm{d} X^{\alpha}}{\mathrm{d} \tau} .
$$

From this action the field equations and the equation of motion for the particle are derived in the usual way and we obtain

$$
\partial_{\mu} E^{\mu}=-S \quad \text { and } \quad \partial_{\mu} E_{\nu}-\partial_{\nu} E_{\mu}=0
$$

with

$$
S(x)=e \int \mathrm{d} \tau \delta_{4}(x-X(\tau))
$$

and

$$
[m-e \phi(X(\tau))]\left(g^{\mu \nu}+u^{\mu} u^{\nu}\right) \dot{u}_{\nu}=e\left(g^{\mu \nu}+u^{\mu} u^{\nu}\right) E_{v} .
$$

In the derivation of this last equation it was important to keep the factor $\sqrt{-g_{\alpha \beta} u^{\alpha} u^{\beta}}$ in the chronometrically invariant Lagrangian (2.3) and only in the very end replace it by $\sqrt{-u^{2}}=1$.

The same equation can be derived from the energy-momentum tensor $T^{\mu \nu}$, as we will now demonstrate. We define the (symmetric) $T^{\mu \nu}$ with the method of variation of the chronometric invariant action $A$ of eq. (2.1) with respect to the metric tensor. [See e.g. ref. 14), p. 360.]

$$
\delta_{g} A=\delta_{8} \int \mathrm{d}_{4} x \sqrt{\bar{g}} \mathscr{L}=\frac{1}{2} \int T^{\mu \nu} \delta g_{\mu \nu} \sqrt{\tilde{g}} \mathrm{~d}_{4} x .
$$

In this method it is important to have all indices in their "natural" positions, i.e., lower indices for $g_{\mu \nu}$ and $E_{\mu}$ and upper indices for $X^{\mu}(\tau)$ and $u^{\mu}$. Keeping 
this in mind and using ${ }^{14}$ )

$$
\delta g^{\mu \nu}=-g^{\mu \lambda} g^{\nu \sigma} \delta g_{\lambda \sigma} \quad \text { and } \quad \delta \tilde{g}=\tilde{g} g^{\mu \nu} \delta g_{\mu \nu},
$$

the energy-momentum tensor becomes

$$
T^{\mu \nu}(x)=E^{\mu} E^{\nu}-\frac{1}{2} g^{\mu \nu} E^{\lambda} E_{\lambda}+\int \mathrm{d} \tau \delta_{4}(x-X(\tau))\left(m_{0}-e \phi(X(\tau))\right) u^{\mu} u^{\nu} .
$$

We now consider the field-plus-interaction part of this tensor

$$
\tilde{T}^{\mu \nu} \equiv E^{\mu} E^{\nu}-\frac{1}{2} g^{\mu \nu} E^{\lambda} E_{\lambda}-e \int \mathrm{d} \tau \delta_{4}(x-X(\tau)) \phi(X(\tau)) u^{\mu} u^{\nu}
$$

and show how it can be used to derive the equation of motion. A simple calculation gives

$$
\partial_{\mu} \tilde{T}^{\mu \nu}=-S E^{\nu}-e \int \mathrm{d} \tau \delta_{4}(x-X(\tau))\left[\dot{u}^{\nu} \phi-E_{\mu} u^{\mu} u^{\nu}\right] .
$$

Since the momentum of the field is given by $\int \mathrm{d}_{3} x \tilde{T}^{0 v}(x, t)$ we can calculate the change in the momentum of the particle from the conservation of momentum and obtain

$$
\frac{\mathrm{d} p^{\nu}}{\mathrm{d} t}=-\int \mathrm{d}_{3} x \partial_{0} \tilde{T}^{0 \nu}=-\int \mathrm{d}_{3} x \partial_{\mu} \tilde{T}^{\mu \nu}
$$

Substitution of (2.12) gives after performing the integrations over $\boldsymbol{x}$ and $\tau$ :

$$
\frac{\mathrm{d} p^{\mu}}{\mathrm{d} \tau}-e \phi \dot{u}^{\mu}=e\left(g^{\mu \nu}+u^{\mu} u^{\nu}\right) E_{v}
$$

By choosing the relation between $p^{\mu}$ and $u^{\mu}$ as

$$
p^{\mu}=m u^{\mu}
$$

it is seen that (2.14) and (2.7) are equivalent, since the equations are consistent with $u^{2}=-1$. In the spin case we will get consistency only if terms $O\left(e^{2}\right)$ in (2.14) can be neglected, i.e., for weak fields. Omitting the same terms here too we obtain for the spinless case as final equation of motion

$$
m \dot{u}^{\mu}=e\left(g^{\mu \nu}+u^{\mu} u^{\nu}\right) E_{\nu}
$$

At this point we want to observe that, although this equation looks rather innocent, it should not be taken too seriously for strong fields or for too long times. This becomes clear by considering the case where a particle is accelerated by a constant field $E_{0}=0, E=(0,0, E)$. The solution of eq. (2.16) is then given by $u^{v}=\left(\sqrt{1+u^{2}}, 0,0, u\right)$ with 


$$
u(\tau)=\operatorname{tg}\left(\frac{e E}{m} \tau\right)
$$

which shows that after a finite eigentime the particle would reach the velocity of light as seen from a fixed frame. This effect is even stronger when $\phi(X(\tau))$ in eq. (2.14) is not neglected. This nonsensical result should eventually be removed by taking radiation damping into account. In the present paper, however, we will not try to do this.

We will now apply the same method to the case where the spin is not zero. The derivation of eq. (2.14) is still valid, but the relation (2.15) between momentum and velocity will have to be modified. Also, we will show that one has to take the constraint $p^{\mu} \sigma_{\mu \nu}=0$ and not $u^{\mu} \sigma_{\mu \nu}=0$. The spin equation is derived from the conservation of angular momentum in the following way.

The density of angular momentum residing in the field plus interaction is equal to

$$
\tilde{J}_{\alpha \beta}^{\mu}(x)=x_{\alpha} \tilde{T}_{\beta}^{\mu}-x_{\beta} \tilde{T}_{\alpha}^{\mu},
$$

where $\tilde{T}^{\mu \nu}$ is given by eq. (2.11). Now consider two spacelike three-dimensional surfaces $S_{1}$ and $S_{2}$ (see fig. 1), which enclose a four-dimensional volume $\Omega$. Let $\tau$ and $\tau+\mathrm{d} \tau$ be the times at which the worldline pierces these surfaces. The increase in the total field-angular momentum $\tilde{J}_{\alpha \beta}=\int \mathrm{d}_{3} x \tilde{J}_{\alpha \beta}$ during this time interval is equal to

$$
\mathrm{d} \tilde{J}_{\alpha \beta}=\int_{S_{2}} \mathrm{~d}_{3} x \tilde{J}_{\alpha \beta}^{0}-\int_{S_{1}} \mathrm{~d}_{3} x \tilde{J}_{\alpha \beta}^{0}=\int_{\Omega} \mathrm{d}_{4} x \partial_{0} \tilde{J}_{\alpha \beta}^{0}=\int_{\Omega} \mathrm{d}_{4} x \partial_{\mu} \tilde{J}_{\alpha \beta}^{\mu} .
$$

Substituting eq. (2.18) and using the symmetry of $\tilde{T}_{\alpha \beta}$ then gives

$$
\mathrm{d} \tilde{J}_{\alpha \beta}=\int_{\Omega} \mathrm{d}_{4} x\left[x_{\alpha} \partial_{\mu} \tilde{T}_{\beta}^{\mu}-x_{\beta} \partial_{\mu} \tilde{T}_{\alpha}^{\mu}\right]
$$

The integration can be performed using the expression (2.12). From the conservation of angular momentum we can now calculate the change in the

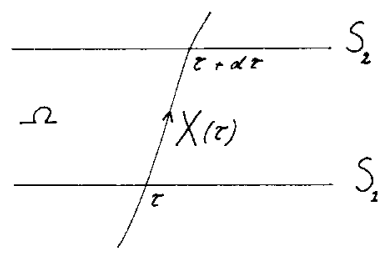

Fig. 1. Worldline for the calculation of $d \tilde{J}_{\alpha \beta}$. 
angular momentum $j_{\alpha \beta}$ of the particle and obtain

$$
\frac{\mathrm{d} j_{\alpha \beta}}{\mathrm{d} \tau}=X_{\alpha}(\tau) \frac{\mathrm{d} p_{\beta}}{\mathrm{d} \tau}-X_{\beta}(\tau) \frac{\mathrm{d} p_{\alpha}}{\mathrm{d} t},
$$

where eq. (2.14) has been used. Taking the orbital angular momentum of the particle as

$$
l_{\alpha \beta}=X_{\alpha} p_{\beta}-X_{\beta} p_{\alpha}
$$

the internal angular momentum becomes $S_{\alpha \beta}=j_{\alpha \beta}-l_{\alpha \beta}$. Since we have no electric dipole moment we can identify this $S_{\alpha \beta}$ with the spin tensor $\sigma_{\alpha \beta}$. The equation for this spin tensor can now be derived from (2.20) and (2.21) and becomes

$$
\dot{\sigma}_{\alpha \beta}=u_{\beta} p_{\alpha}-u_{\alpha} p_{\beta} .
$$

This equation, together with the one obtained from (2.14) by neglecting the term $e \phi \dot{u}^{\mu}$, i.e.,

$$
\dot{p}^{\mu}=e\left(g^{\mu \nu}+u^{\mu} u^{\nu}\right) E_{\nu}
$$

determines the motion of the particle with its spin, as soon as we have established a relation between the momentum of the particle and its velocity.

If we had a Lagrangian it would contain scalars formed by contracting $u$ with internal variables related to the spin. Then, $p$ would no longer be proportional to $u$. We anticipate this by writing

$$
p^{\mu}=m_{0}(\tau) u^{\mu}+v^{\mu},
$$

and solve for $v^{\mu}$ and for $m_{0}(\tau)$. We demand that in regions where the external field is zero $v$ must be zero and $m_{0}$ must be $m$, where $p^{2}=-m^{2}$.

In solving for $v$ there are two distinct ways to proceed. In regions where the external field is nonzero we may require either

$$
\begin{aligned}
& \sigma_{\alpha \beta} u^{\beta}=0, \quad \text { or } \\
& \sigma_{\alpha \beta} p^{\beta}=0 .
\end{aligned}
$$

In regions with no external field (1.2b) reduces to (1.2a) as $v$ is to be zero in such regions. Let us investigate both possibilities (1.2a) and (1.2b). We shall see that the former leads to undesirable equations after which we proceed with the latter.

Assuming (1.2a), the vector $v$ can be determined uniquely from (2.22), the result is

$$
v_{\alpha}=\sigma_{\alpha \beta} \dot{u}^{\beta}=-\dot{\sigma}_{\alpha \beta} u^{\beta} .
$$

Substituting into (2.22) and (2.23) gives the equations 


$$
\begin{aligned}
& \dot{\sigma}_{\alpha \beta}=\sigma_{\alpha \gamma} \dot{u}^{\gamma} u_{\beta}-\sigma_{\beta \gamma} \dot{u}^{\gamma} u_{\alpha}, \\
& \left(m_{0} \dot{u}^{\alpha}+\dot{m}_{0} u^{\alpha}+\sigma^{\alpha \beta} \ddot{u}_{\beta}\right)=e\left(g^{\alpha \beta}+u^{\alpha} u^{\beta}\right) E_{\beta} .
\end{aligned}
$$

Equations (2.26), (2.27) have third order derivatives of the coordinates. Also $v$ given by (2.25) is not zero outside the region of interaction. Setting $E_{\beta}=0$ and $m_{0}=m$ in (2.27) gives Frenkel's equation ${ }^{8}$ ) for a free particle. These equations have solutions which give a helical motion for a free particle ${ }^{13}$ ). The equations are, however, consistent with the conservation laws as is also obvious from the fact that they can be derived from a Lagrangian ${ }^{8}$ ). We conclude that the equations (2.26), (2.27) are undesirable and from here on we shall work with (1.2b), also in the case of the electromagnetic field. One could limit oneself to solutions which originally have a radius zero for the helical motion. This radius would not be disturbed much by a weak external field and similarly $v^{\mu}$ would be small in such a field. However, $v^{\mu}$ will not always return to zero when the particle leaves the field and a transit through a strong field will sometimes lead to a free particle with a large helical motion.

We now demand that

$$
p^{\mu} \sigma_{\mu \nu}=0
$$

for all times. From this follows

$$
\dot{p}^{\mu} \sigma_{\mu \nu}+p^{\mu} \dot{\sigma}_{\mu \nu}=0 .
$$

Writing (2.22) as

$$
\dot{\sigma}_{\mu \nu}=\frac{1}{m_{0}}\left(v_{\mu} p_{\nu}-v_{\nu} p_{\mu}\right)
$$

and substituting this into (2.29) gives

$$
\dot{p}^{\mu} \sigma_{\mu \nu}+\frac{1}{m_{0}} p^{\mu}\left(v_{\mu} p_{\nu}-v_{\nu} p_{\mu}\right)=0 .
$$

Without loss of generality we may assume $v$ to be perpendicular to $p$, as a component in the direction of $p$ can be taken care of via $m_{0}$ in (2.24). Hence we set

$$
p^{\mu} v_{\mu}=0
$$

and find

$$
v_{\nu}=\frac{m_{0}}{p^{2}} \dot{p}^{\mu} \sigma_{\mu \nu}
$$

From the anti-symmetry of $\sigma_{\mu \nu}$ then follows that

$$
v_{\nu} \dot{p}^{v}=0 .
$$


Combining this with

$$
u_{\nu} \dot{p}^{\nu}=0,
$$

which is derived from (2.23), we immediately obtain $p_{v} \dot{p}^{\nu}=0$, so that $p^{2}=$ $-m^{2}$ is indeed a constant. Also

$$
\sigma^{2}=\sigma^{\mu \nu} \sigma_{\mu \nu}=\text { constant, }
$$

because of (2.30) and (2.28). Eq. (2.33) can now be written as

$$
v_{\nu}=-\frac{e m_{0}}{m^{2}}\left(g^{\mu \lambda}+u^{\mu} u^{\lambda}\right) E_{\lambda} \sigma_{\mu \nu}
$$

and we observe that this is of first order in $e$. Since

$$
u^{\mu} \sigma_{\mu \nu}=\frac{1}{m_{0}}\left(p^{\mu}-v^{\mu}\right) \sigma_{\mu \nu}=-\frac{1}{m_{0}} v^{\mu} \sigma_{\mu \nu}=\mathrm{O}(e),
$$

we can write (2.37)

$$
v_{\nu}=-\frac{e m_{0}}{m^{2}} E^{\mu} \sigma_{\mu \nu}+\mathrm{O}\left(e^{2}\right)
$$

The function $m_{0}(\tau)$ can be determined by taking the square of $p^{\mu}$ and using (2.24). We find

$$
m_{0}^{2}=m^{2}-v^{2} \text {. }
$$

On substitution of (2.39) we can solve for $m_{0}$ :

$$
m_{0}(\tau)=m\left[1-\frac{e^{2}}{2 m^{4}}\left(E^{\alpha} \sigma_{\alpha \gamma}\right)\left(E_{\beta} \sigma^{\beta \gamma}\right)\right]+\mathrm{O}\left(e^{3}\right),
$$

so that eq. (2.39) becomes

$$
v_{\nu}=-\frac{e}{m} E^{\mu} \sigma_{\mu \nu}+\mathrm{O}\left(e^{2}\right) .
$$

This can now be used in the equations (2.23) and (2.30) to give

$$
\dot{u}^{\mu}=\frac{e}{m}\left(g^{\mu \nu}+u^{\mu} u^{\nu}\right) E_{\nu}+\frac{e}{m^{2}} u^{\lambda} \partial_{\lambda} E_{\nu} \sigma^{\nu \mu}
$$

and

$$
\dot{\sigma}_{\mu \nu}=\frac{e}{m}\left(\sigma_{\mu \lambda} u_{\nu}-\sigma_{\nu \lambda} u_{\mu}\right) E^{\lambda}
$$

In both equations terms of order $e^{2}$ have been neglected. Using the definition (1.5b) for the spinvector the last two equations can also be written as 


$$
\dot{u}^{\mu}=\frac{e}{m}\left(g^{\mu \nu}+u^{\mu} u^{\nu}\right) E_{\nu}-\frac{e}{m^{2}} \epsilon^{\mu \nu \lambda \sigma} W_{\lambda} u_{\sigma} u^{\alpha} \partial_{\alpha} E_{\nu}
$$

and

$$
\dot{W}^{\mu}=\frac{e}{m}\left(W^{\nu} E_{\nu}\right) u^{\mu} .
$$

They are consistent with $u^{2}=-1, W^{2}=$ constant and $u^{\mu} W_{\mu}=0$. Note also that these equations differ from those given by Schwinger"I) in the term in (2.45) containing the derivatives of the field. This term, however, is necessary in order to satisfy the conservation laws.

\section{Spinning particle in an electromagnetic field}

First, consider a particle without spin, interacting with the electromagnetic field. The Lagrange density is

$$
\mathscr{L}=-{ }_{4}^{1} F_{\mu \nu}(x) F_{\alpha \beta}(x) g^{\mu \alpha} g^{\nu \beta}+e \int \mathrm{d} \tau \tilde{g}^{-1 / 2} \delta(x-X(\tau)) A_{\mu}(X(\tau)) u^{\mu},
$$

where we have omitted the part describing the free particle. With the action given by (3.1) one can repeat the analysis of the beginning of section 2 . We limit ourselves to giving some of the formulae needed in the remainder of this section. The Maxwell equations with source are

$$
\begin{aligned}
& \partial_{\mu} F^{\mu \nu}(x)=-j^{\nu}(x) \\
& \partial_{\alpha} F_{\beta \gamma}+\partial_{\beta} F_{\gamma \alpha}+\partial_{\gamma} F_{\alpha \beta}=0,
\end{aligned}
$$

where

$$
j^{\nu}(x)=j_{(0)}^{\nu}(x)=e \int \mathrm{d} \tau \delta(x-X(\tau)) u^{\mu}(\tau)
$$

The field $F^{\mu \nu}$ and its dual $\tilde{F}^{\mu \nu}=\frac{1}{2} \epsilon^{\mu \nu \lambda \sigma} F_{\lambda \sigma}$ are related to the electric and magnetic fields in the usual way

$$
F^{0 k}=E_{k} ; \quad F^{k l}=\epsilon_{k l m} B_{m} ; \quad \tilde{F}^{0 k}=B_{k} ; \quad \tilde{F}^{k l}=-\epsilon_{k l m} E_{m} .
$$

As $j^{\nu}$ has its index up and $A_{\nu}$ its index down, there is- no interaction contribution to the energy momentum tensor. For this tensor of Maxwell field (+ interaction) one finds

$$
\tilde{T}_{(0)}^{\mu \nu}(x)=F_{\alpha}^{\mu} F^{\nu \alpha}-\frac{1}{4} F^{\alpha \beta} F_{\alpha \beta} g^{\mu \nu} .
$$

With (3.2), (3.3), one has

$$
\partial_{\mu} \tilde{T}_{(0)}^{\mu \nu}(x)=-F^{\nu \mu} \mathrm{j}_{\mu}(x) \text {. }
$$


Next, consider a particle with spin. Although we do not have a Lagrangian density for the entire system, we assume a Lagrangian density for the Maxwell field interacting with its sources. In this Lagrangian density we now expect a possible gyromagnetic interaction term of the form

$$
\frac{-e g}{4 m} \int \mathrm{d} \tau \tilde{g}^{-1 / 2} \delta(x-X(\tau)) \sqrt{-u^{\alpha} u^{\beta} g_{\alpha \beta}}(F \cdot \sigma),
$$

where $F \cdot \sigma=\sigma^{\mu \nu}(\tau) F_{\mu \nu}(X(\tau))$. Because of symmetry arguments such a coupling was not possible to the scalar field of section 2 . We have used $g$ to denote the gyromagnetic ratio. The factor involving the square root is necessary in order to maintain chronometric invariance, which will eventually ensure that $p^{2}$ is a constant. The indices in (3.7) have been put in their natural places, $\sigma^{\mu \nu}$ being an angular momentum, has both indices up. The term (3.7) gives a contribution to the energy momentum tensor $\tilde{T}^{\mu \nu}$ for Maxwell field plus interaction. We get

$$
\begin{aligned}
\tilde{T}^{\mu \nu}(x)= & F_{\alpha}^{\mu}(x) F^{\nu \alpha}(x)-{ }_{4}^{1} g^{\mu \nu} F_{\alpha \beta} F^{\alpha \beta}(x) \\
& +\frac{e g}{4 m} \int \mathrm{d} \tau \delta(x-X(\tau)) \sigma^{\alpha \beta}(\tau) F_{\alpha \beta}(X(\tau)) u^{\mu}(\tau) u^{\nu}(\tau) .
\end{aligned}
$$

It is useful to note that, via a partial integration, the interaction term (3.7) is equivalent to a modification of the current (3.4):

$$
j^{\mu}(x)=e \int \mathrm{d} \tau\left\{u^{\mu}(\tau) \delta(x-X(\tau))+\frac{g}{2 m} \sqrt{-u^{2}} \sigma^{\mu \alpha}(\tau) \partial_{\alpha} \delta(x-X(\tau))\right\} .
$$

With (3.9) the Maxwell equations can be maintained in the form (3.2), (3.3). Thus, one finds

$$
\begin{aligned}
\partial_{\mu} \tilde{T}^{\mu \nu}(x)= & -F^{\nu \mu} j_{\mu}(x) \\
& +\frac{e g}{4 m} \int \mathrm{d} \tau \delta(x-X(\tau))\left\{\dot{u}^{\nu}(F \cdot \sigma)+u^{\nu} \frac{\mathrm{d}}{\mathrm{d} \tau}(F \cdot \sigma)\right\} .
\end{aligned}
$$

From the conservation laws of total linear four momentum and four angular momentum, one obtains in a way analogous to that explained in detail in section 2

$$
\dot{p}^{\mu}=e F^{\mu \beta} u_{\beta}+\frac{e g}{2 m} \sigma_{\alpha \beta} \partial^{\beta} F^{\mu \alpha}-\frac{e g}{4 m}\left\{\dot{u}^{\mu}(F \cdot \sigma)+u^{\mu}(\dot{F} \cdot \sigma)+u^{\mu}(F \cdot \dot{\sigma})\right\} .
$$

With (3.3) the second term on the right-hand side of this last equation can be written as $(-e g / 4 m) \partial^{\mu}(F \cdot \sigma)$. It can then be combined with the fourth term 
giving

$$
\dot{p}^{\mu}=e F^{\mu \beta} u_{\beta}-\frac{e g}{4 m}\left(g^{\mu \beta}+u^{\mu} u^{\beta}\right) \partial_{\beta}(F \cdot \sigma)-\frac{e g}{4 m} \dot{u}^{\mu}(F \cdot \sigma)-\frac{e g}{4 m} u^{\mu}(F \cdot \dot{\sigma}) .
$$

The last two terms are $\mathrm{O}\left(e^{2}\right)$ and will be omitted in what follows.

For the spin equation we obtain, again from the conservation law of total angular four momentum:

$$
\dot{\sigma}_{\mu \nu}=p_{\mu} u_{\nu}-p_{\nu} u_{\mu}+\frac{e g}{2 m}\left(F_{\mu}^{\cdot \beta} \sigma_{\beta \nu}-F_{\nu}^{\cdot \beta} \sigma_{\beta \nu}\right)
$$

There is a difference with the spin zero field in that the current (3.9) contains derivatives of the delta function. These derivatives act on the $x_{\alpha}$ and $x_{\beta}$ on the right-hand side of (2.19) and produce the last term on the right-hand side of (3.12).

Before proceeding with our derivation let us point out that (3.11) and (3.12) follow uniquely from the conservation laws and from the expression (3.9) for $j^{\mu}(x)$. All what follows is unique once $j^{\mu}(x)$ is given. Therefore it is a natural question to ask what is the most general expression for $j^{\mu}$. Because of (3.2) the general $j^{\mu}$ must satisfy $\partial_{\mu} j^{\mu}=0$ as an identity. Also we want it to be local and not to contain derivatives of the delta function of order higher than one. Furthermore, it should be constructed out of $u$ and $\sigma$ and should not contain the fields. We have $u^{2}=-1, \sigma^{2}=$ constant and $\sigma_{\mu \nu} p^{\nu}=0$, which implies with (2.24) and with $v$ linear in the fields, that also $u^{\mu} \sigma_{\mu \nu}$ may be regarded as zero in constructing $j^{\mu}$. Using all this information one can show that (3.9) is the most general expression for $j^{\mu}$. For the scalar field the source term $(S(x)$ of eq. (2.6) also had to be a scalar quantity and could therefore not be generalized to include the spin variables.

For the same reasons as in section 2 we do not expect $p$ and $u$ to be parallel. We therefore write again

$$
p^{\mu}=m_{0}(\tau) u^{\mu}+v^{\mu}
$$

Demanding (1.2b), i.e., $\sigma \cdot p=0$, then gives

$$
v_{\beta}=\frac{m_{0}}{p^{2}}\left\{\dot{p}^{\alpha} \sigma_{\alpha \beta}+\frac{e g}{2 m} p_{\mu} F^{\mu v} \sigma_{\nu \beta}\right\}=\mathrm{O}(e),
$$

so that

$$
v_{\beta} \dot{p}^{\beta}=\mathrm{O}\left(\mathrm{e}^{2}\right) .
$$

Therefore

$$
\frac{\mathrm{d}}{\mathrm{d} \tau} p^{2}=2 p_{\beta} \dot{p}^{\beta}=2\left(m_{0} u_{\beta}+v_{\beta}\right) \dot{p}^{\beta}=\mathrm{O}\left(e^{2}\right),
$$


where the last equality sign follows from (3.14) and from $u_{\beta} \dot{p}^{\beta}=0$, which is obvious from (3.11).

So we obtain again that

$$
p^{2}=-m^{2}=\text { constant }+\mathrm{O}\left(e^{2}\right) .
$$

The $\mathrm{O}\left(e^{2}\right)$ terms are zero for $g=0$. The square of the spin is constant, even for $g \neq 0$. This is so because from (3.12) it follows that

$$
\frac{\mathrm{d}}{\mathrm{d} \tau} \sigma^{2}=-\frac{2 e g}{m} \sigma^{\alpha \beta} F_{\beta \gamma} \sigma_{\beta}^{\gamma}=0 .
$$

The last equality is a consequence of the anti-symmetry of $F$ and $\sigma$. As in (2.41) we find again that $m_{0}=m+\mathrm{O}\left(e^{2}\right)$. From (3.13) and (3.11) $v_{\beta}$ becomes

$$
v_{\beta}=-\frac{e}{2 m}(g-2) u_{\mu} F^{\mu \nu} \sigma_{\nu \beta}+\frac{e g}{4 m^{2}}\left(g^{\mu \nu}+u^{\mu} u^{\nu}\right) \partial_{\nu} F_{\lambda \rho} \sigma^{\lambda \rho} \sigma_{\mu \beta}
$$

and its derivative

$$
\dot{v}_{\beta}=-\frac{e}{2 m}(g-2) u_{\mu} u_{\lambda} \partial^{\lambda} F^{\mu v} \sigma_{\nu \beta},
$$

in which not only terms $\mathrm{O}\left(e^{2}\right)$, but also terms containing second derivatives of the fields are neglected.

The expressions (3.18) and (3.19) for $v_{\beta}$ and $\dot{v}_{\beta}$ are now substituted into (3.11) and (3.12) and in this way we find the most general equations satisfying our original conditions:

$$
\dot{u}^{\mu}=\frac{e}{m} F^{\mu \nu} u_{\nu}-\frac{e g}{4 m^{2}}\left(g^{\mu \nu}+u^{\mu} u^{\nu}\right) \partial_{\nu} F_{\alpha \beta} \sigma^{\alpha \beta}+\frac{e}{2 m^{2}}(g-2) u^{\alpha} u^{\beta} \partial_{\beta} F_{\alpha \nu} \sigma^{\nu \mu}
$$

and

$$
\begin{aligned}
\dot{\sigma}_{\alpha \beta}= & \frac{e g}{2 m}\left(F_{\alpha \mu} \sigma_{\cdot \beta}^{\mu}-F_{\beta \mu} \sigma_{\cdot \alpha}^{\mu}\right. \\
& +\frac{e}{2 m}\left[(g-2) u_{\nu} F^{\nu \mu}-\frac{g}{2 m} \partial^{\mu} F^{\lambda \rho} \sigma_{\lambda \rho}\right]\left[u_{\alpha} \sigma_{\mu \beta}-u_{\beta} \sigma_{\mu \alpha}\right] .
\end{aligned}
$$

Using eqs. (1.5b) and (1.6b) the spin tensor in these equations can be replaced by the spin vector and the equations then become

$$
\begin{array}{r}
\dot{u}^{\mu}=\frac{e}{m} F^{\mu \nu} u_{v}-\frac{e g}{2 m^{2}}\left(g^{\mu \nu}+u^{\mu} u^{\nu}\right) \partial_{\nu} \tilde{F}^{\rho \lambda} W_{\rho} u_{\lambda} \\
+\frac{e}{2 m^{2}}(g-2)\left(g^{\mu \nu}+u^{\mu} u^{\nu}\right) u^{\alpha} \partial_{\alpha} \tilde{F}_{\nu \beta} W^{\beta}
\end{array}
$$


and

$$
\dot{W}^{\mu}=\frac{e g}{2 m} F^{\mu \nu} W_{\nu}+\frac{e}{2 m}(g-2)\left(u^{\alpha} F_{\alpha \beta} W^{\beta}\right) u^{\mu}-\frac{e g}{2 m^{2}}\left(W^{\alpha} \partial_{\alpha} \tilde{F}_{\beta \gamma} W^{\beta} u^{\gamma}\right) u^{\mu}
$$

Also these equations are consistent with $u^{2}=-1, W^{2}=$ constant and $u \cdot W=$ 0 . If we disregard for a moment the $(g-2)$ term in (3.22), the last two equations are identical with eqs. (15) and (16) of the paper by Good ${ }^{12}$ ), when his quadrupole terms are omitted.

The existence of the $(g-2)$ term in (3.22) was pointed out before in ref. 1 . In the instantaneous rest system it is equal to

$$
-\frac{e}{2 m^{2}}(g-2) S \wedge \frac{\partial E}{\partial t}
$$

Although this term is small compared to the Lorentz force, its presence is dictated by the conservation laws and it would be nice if it could be observed.

Neglecting in (3.22) and (3.23) all terms which contain derivatives of $F_{\mu \nu}$, one obtains

$$
m \dot{u}^{\mu}=e F^{\mu \nu} u_{\nu}
$$

and

$$
\dot{W}^{\mu}=\frac{e g}{2 m} F^{\mu \nu} W_{\nu}+\frac{e}{2 m}(g-2)\left(u^{\alpha} F_{\alpha \beta} W^{\beta}\right) u^{\mu},
$$

which are the equations postulated by Bargmann et al. ${ }^{5}$ ). These equations are, up to the order considered, consistent with the conservation laws as we have shown. However, by neglecting derivatives of the field these equations do not contain the Stern-Gerlach effect.

Note, that similar to what happened in section 2 for the scalar field, the $\sqrt{-u^{2}}$ term in (3.9), to which we were guided by chronometric invariance, is essential to ensure (1.3) up to order $e^{2}$. in this respect our work differs from that of ref. 1 .

Let us also remark that we could have made assumption (1.2a) instead of (1.2b) also for the interaction with the Maxwell field. This is essentially what was done by Frenkel ${ }^{8}$ ) and leads to his equations. These equations, however, contain third derivatives of the position coordinates and do not satisfy the condition that $p$ and $u$ always be parallel outside the fields (helical motion). One could take solutions were initially $p$ and $u$ are parallel. Weak fields would not disturb this much, but $v$, the difference between $p$ and $u$, would in general not return to zero, but be of first order in the fields which the particle has 
traversed. However after going through a strong field one would, in general, obtain particles with a large $v$ and with a large helical motion.

Finally, our eqns. (3.20) and (3.21) are identical to (61) and (62) of ref. 1, when substituting $m$ for their $M^{*}$. The derivation which leads to the final equation is, however, somewhat different. Just like in the scalar case we did see to it that chronometric invariance is satisfied at all stages. Because of this chronometric invariance there is a difference in the equation for $\dot{p}$. Compare their equation (52) to our (3.11), which contains a projection operator, just as it occurred in our equation (2.23) for the scalar field. Thus, for us it is possible to maintain $p^{2}=-m^{2}$ at all stages, with $m^{2}$ constant (up to second order in $e$ ). The authors of ref. 1 had, however, $p^{2}=-M^{2}$ not constant, and had to switch to the quantity $M^{*}$, which is then also conserved (up to second order in $e$ ).

\section{Conclusions}

Assuming that there is an action and a Lagrangian density describing the external field including the particle as a source, we use the overall conservation laws of linear and of angular momentum to derive the equations of motion of spinning particles. In this derivation the only assumptions made are the following. First one makes an assumption about the source term by which the particle acts back on the fields. By arguments of Poincaré invariance, by demanding that the source terms do not include the fields and by requiring chronometric invariance (invariance for reparametrizing the worldline of the particle) we find essentially unique expressions for these source or interaction terms. Second, one makes the assumption (1.2b) that $p \cdot \sigma=0$. This differs from $u \cdot \sigma=0$ only in regions where the external field does not vanish. This follows from our third assumption, which is that $p$ and $u$ be parallel outside the fields. This third assumption can be checked after our derivation, as was done in sections 2 and 3. We also obtain $\mathrm{d} p^{2} / \mathrm{d} \tau=0, \mathrm{~d} \sigma^{2} / \mathrm{d} \tau=0$ and $\mathrm{d} u^{2} / \mathrm{d} \tau=0$.

The final equations obtained are unique. They differ somewhat from those of $\operatorname{Good}^{12}$ ), which were derived without explicit consideration of conservation laws. The final equations agree with those of Suttorp and de Groot. There is, however, an essential difference in the derivation, which was discussed at the end of section 3 .

\section{Acknowledgement}

The authors thank Prof. E.P. Wigner who made a number of remarks which started this work. 


\section{References}

1) L.G. Suttorp and S.R. de Groot, Nuovo Cim. 65 (1970) 245.

2) P. Nyborg, Nuovo Cim. 23 (1962) 47, 26 (1962) 821.

3) W.G. Dixon, Nuovo Cim. 38 (1965) 1616, 34 (1964) 317.

4) See e.g. N.F. Mott and H.S.W. Massey, The Theory of Atomic Collisions (Oxford U.P., Oxford, 1965) p. 214.

5) V. Bargmann, L. Michel and V.L. Telegdi, Phys. Rev. Lett. 2 (1959) 435. See Also M. Fierz and V.L. Telegdi in: Quanta (The University of Chicago Press, Chicago, 1970) p. 196.

6) See ref. 4 , p. 215.

7) A.J. Hanson and T. Regge, Ann. Phys., N.Y. 87 (1974) 498;

N. Mukunda, H. van Dam and L.C. Biedenharn, Duke preprint, March 1980.

8) J. Frenkel, Z. Phys. 37 (1926) 243.

9) M.H.L. Pryce, Proc. Roy. Soc. A195 (1948) 62.

10) P. Grassberger, J. Phys. A: Math. Gen. 11 (1978) 1221.

11) J. Schwinger, Am. J. Phys. 42 (1973) 510.

12) R. H. Good, Phys. Rev. 125 (1962) 2112.

13) J. Weyssenhoff and A. Raabe, Acta Phys. Polon. 9 (1947) 7.

14) See e.g. S. Weinberg, Gravitation and Cosmology (Wiley, New York, 1972) p. 47. 